\title{
Eosinophilia and symptomatic pulmonary amyloidosis
}

\section{A A Haydar, A Li, R Hilton, B Lams, R Hangartner, M Weber, D J A Goldsmith}

Postgrad Med J 2004;80:738-739. doi: 10.1136/pgmj.2003.013052

The case of a man with progressive breathlessness and pulmonary infiltration caused by $\mathrm{AL}$ amyloidosis associated with multiple myeloma is presented. There was a marked peripheral eosinophilia, which has not previously been described with amyloidosis.

E osinophilia is a challenging disease with a wide differential diagnosis (see box 1). A broad variety of allergic, infectious, neoplastic, and idiopathic diseases are associated with increased blood and/or tissue eosinophilia and range in severity from self limited conditions to life threatening disorders. We report a patient who had marked peripheral eosinophilia associated with pulmonary symptoms. He was diagnosed to have pulmonary amyloidosis.

\section{CASE REPORT}

A 72 year old man presented with a serum creatinine concentration of $300 \mu \mathrm{mol} / \mathrm{l}$, haemoglobin $97 \mathrm{~g} / \mathrm{l}$, and hypercalcaemia. Renal ultrasound revealed two small kidneys with thin cortices; 24 hour urine protein was 3.4 g. A kidney biopsy specimen showed focal scarring of the interstitium only, and some fractured renal tubular casts. Congo red staining was negative. Urine immunoelectrophoresis showed free lambda light chains with a low plasma immunoglobulin level. Bone marrow trephine biopsy confirmed the presence of $25 \%$ plasmacytosis consistent with multiple myeloma.

Chemotherapy was started with cyclophosphamide, dexamethasone, and pamidronate. Progression to end stage renal failure was rapid, necessitating continuous ambulatory peritoneal dialysis and cessation of chemotherapy.

Two years later, the patient was admitted with a month's history of progressive dyspnoea. Examination revealed bibasilar crackles and a friction rub. Chest radiography showed bilateral lower zone infiltrates and arterial blood gases on room oxygen demonstrated a partial pressure of oxygen of $7.6 \mathrm{kPa}$ and partial pressure of carbon dioxide of $5.5 \mathrm{kPa}$. The white cell count was $10 \times 10^{9} / \mathrm{l}$ with an eosinophilia of $35 \%$ (normal previously); the absolute eosinophil count was $3500 \mathrm{cell} / \mu \mathrm{l}$. High resolution computed tomography of the chest showed ground glass opacities in both lungs with confluent nodules on the right. Pulmonary function tests demonstrated a restrictive pattern (forced expiratory volume in one second $=1.18 \mathrm{l}$, forced vital capacity $=1.33 \mathrm{l}$ ) and impaired gas exchange. Bronchoscopy showed normal mucosa. Cytology revealed eosinophils. There was no evidence of fungi, mycobacterium, parasites, cytomegalovirus, or Pneumocystis carinii pneumonia. Autoantibody screen and complement (C3, C4) were all normal and strongyloides ELISA antibodies negative.

The working diagnosis was acute eosinophilic pneumonia and $60 \mathrm{mg}$ daily oral prednisolone was started and continued (at variable doses) until his death eight month's later. Peripheral eosinophilia resolved completely after a few days, but the patient's dyspnoea improved only marginally. A few weeks later, the patient was readmitted with a significant respiratory deterioration. There was no peripheral eosinophilia. High resolution computed tomography of the chest demonstrated calcified pleural plaques, irregular consolidation in the right lower lobe, and widespread ground glass opacification (see fig 1). Bronchoscopy was normal and polymorphonuclear leucocytes were seen on bronchoalveolar lavage; there was no evidence of infectious organisms.

Attempted transbronchial biopsy (no lung parenchyma) showed normal bronchial mucosa. Open lung biopsy revealed widespread homogenous deposits in alveolar walls, bronchioles and pulmonary vessels, confirmed as amyloidosis by Congo red staining. The patient became progressively more breathless and died shortly after.

\section{DISCUSSION}

The patient presented with peripheral eosinophilia and pulmonary amyloidosis. The cause of the peripheral

Box 1: Eosinophil associated diseases and disorders

\section{Allergic diseases}

- Atopic and related diseases.

- Medication related eosinophilia.

Infectious diseases

- Parasitic infections mostly with helminths.

- Special fungal infection.

Haematological and neoplastic disorders

- Hypereosinophilic syndrome.

- Leukaemia.

- Lymphoma.

- Tumour associated.

- Mastocytosis.

Diseases with specific organ involvement

- Skin and subcutaneous diseases.

- Pulmonary diseases.

- Gastrointestinal diseases.

- Neurological diseases.

- Rheumatological diseases.

- Cardiac diseases.

- Renal diseases.

Immunological reactions

- Specific immune deficiencies.

- Transplant rejection.

Endocrine

- Hypoadrenalism. 


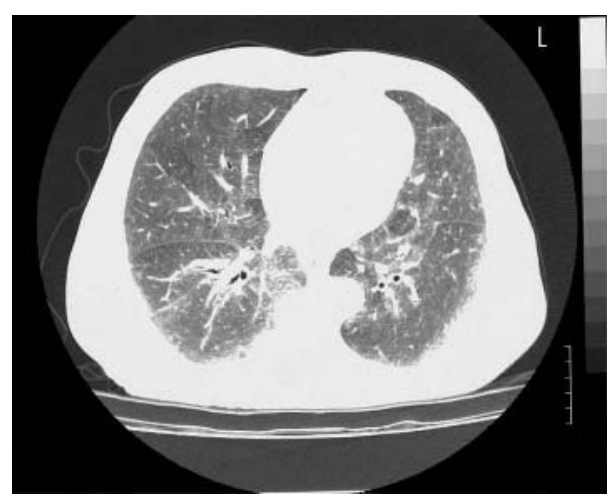

Figure 1 High resolution computed tomogram of the chest showing ground glass opacities in both lungs with confluent nodules on the right.

eosinophilia was investigated thoroughly. History and physical examination ruled out most of the differential diagnoses listed in box 1 (transplant rejection, rheumatological and gastrointestinal diseases). The patient was given a course of steroids and his eosinophilia disappeared but not his chest symptoms.

Pulmonary amyloidosis was diagnosed as the cause of the patient's progressive lung disease, and was confirmed with Congo red staining on tissue biopsy. Amyloidosis, whether disseminated or localised in the lung, has not been reported before as associated with peripheral eosinophilia. Amyloidosis is known to affect virtually any organ system except for the brain. In postmortem studies of amyloid patients, the lungs can be affected by amyloidosis-usually the primary/ multiple myeloma $(\mathrm{AL})$, rather than the secondary (AA) type ${ }^{1}$-but symptomatic pulmonary syndromes are much rarer. Pulmonary amyloidosis is usually a silent disease with scanty respiratory signs; most of the patient symptoms are derived from amyloid infiltration of the heart and kidneys. Pulmonary function testing sometimes not always reveals a restrictive pattern. ${ }^{2}$

Pulmonary involvement is not a major contributor to death in AL amyloidosis, ${ }^{2}$ and the median patient survival with clinically overt lung deposition is about 16 months. The disease of our patient was not classical of pulmonary amyloidosis (asymptomatic and indolent). On the contrary the patient's main symptoms were respiratory in nature with peripheral eosinophilia and his disease progressed rapidly and he died of respiratory failure.
Learning points

- Differential diagnosis of peripheral eosinophilia.

- To learn more about pulmonary amyloidosis: aetiology, different types, radiological appearance, treatment, and prognosis.

An association of eosinophilia and amyloidosis has not previously been reported. By the time a diagnostic lung biopsy was obtained the patient had been on oral steroids for some months, and the peripheral eosinophilia had resolved; no eosinophilic lung infiltration was noted on the lung biopsy, which was remarkable for extensive amyloidosis.

The only therapy that might have been effective for the patient is chemotherapy for primary amyloidosis (for example, melphalan and prednisone). Patients who have a chemotherapeutic response may have an extended survival. ${ }^{3}$ However no controlled trials comparing different chemotherapeutic regimens in pulmonary amyloidosis are available. The patient's condition deteriorated quickly and in view of his frailty it was felt he would not withstand chemotherapy.

\section{Authors' affiliations}

A A Haydar, A Li, R Hilton, D J A Goldsmith, Renal and Transplantation Unit, Guy's and St Thomas' Hospitals, London, UK

B Lams, Chest Medicine, Guy's and St Thomas' Hospitals, London, UK R Hangartner, M Weber, Histopathology, Guy's and St Thomas' Hospitals, London, UK

Correspondence to: Dr David J A Goldsmith, Guy's Hospital, London SE1 9RT, UK; david.goldsmith@gstt.sthames.nhs.uk

Submitted 25 July 2003

Accepted 29 August 2003

\section{REFERENCES}

1 Sculz C, Hauck RW, Nathrath WB, et al. Combined amyloidosis of the upper and lower respiratory tract. Respiration 1995;62:163-6.

2 Cordier JF, Loire R, Brune J. Amyloidosis of the lower respiratory tract. Clinical and pathological features in a series of 21 patients. Chest 1986;90:827-31.

3 Kyle RA, Gertz MA, Greipp PR, et al. A trial of three regimens for primary amyloidosis: colchicine alone, melphalan and prednisone, and melphalan, prednisone, and colchicine. N Engl J Med 1997;336:1202. 